\title{
ASRTS
}

Ocorrência de espécies de caramujos marinhos (Mollusca; Gastropoda) em costões rochosos das praias da Viola e Prainha, na Ilha de Itacuruçá, Rio de Janeiro, Brasil

\author{
Fabio Moraes da Costa $^{1}$, Fabiano Paschoal ${ }^{1}$, Alessandra Araujo de Alcantara ${ }^{2}$, Flavia Cristina dos Santos \\ Rangel ${ }^{2}$
}

\section{AUTHOR AFILIATIONS}

1 Universidade Castelo Branco, Escola da Saúde e Meio Ambiente. Rio de Janeiro, RJ, Brasil.

${ }^{2}$ Laboratório de Biologia Marinha. Centro de Pesquisas Biológicas, Universidade Castelo Branco (UCB), Rio de Janeiro, RJ, Brasil.

\section{CONTACT}

fmcosta@castelobranco.br. paschoalfabiano@gmail.com alessandraalcant@gmail.com

\begin{abstract}
The phylum Mollusca constitutes one of the most diverse groups in forms and species with functional and environmental adaptations specific to all habitats. Gastropods constitute the largest class of the phylum Mollusca, with three subclass: Prosobranchia, Opstobranchia and Pulmonata. The study was carried out with the objective of verifying the occurrence of marine snails (Mollusca; Gastropoda) in the infralittoral zone of the rocky shores of the beaches of Viola and Prainha, Itacuruçá Island, in Rio de Janeiro, Brazil. The samples were collected between the years 2014 and 2016 in a seasonal period, summer, with a total of 15 sample collections and totaling 4 hours of daily activity, with free diving. Seven species were identified: Tegula viridula (GMELIN, 1791); Haemastoma stramonite (LINNAEUS, 1767); Rustica stramanita (LAMARCK, 1822); Neritina virginea (LINNAEUS, 1758); Calliostoma adspersum (PHILIPPI, 1851); Latirus ogum (PETUCH, 1979) and Fissurella clenchi (FARFANTE, 1943). The Viola beach showed the highest incidence of species, totaling 137 specimens. The species $T$. viridula had the highest occurrence in the two areas studied. L. ogum was the species with the lowest occurrence for the study.
\end{abstract}

Keywords: Mollusca, Probranchia, Stramonita haemastoma.

\section{RESUMO}

O filo Mollusca constitui um dos grupos mais diversos em formas e espécies com adaptações funcionais e ambientais específicas para todos os habitats. Os gastrópodes constituem a maior classe do filo Mollusca, apresentando três subclasses: Prosobranchia, Opstobranchia e Pulmonata. O estudo foi realizado com o objetivo de verificar a ocorrência de caramujos-marinhos (Mollusca; Gastropoda) na zona infralitoral dos costões rochosos das praias da Viola e Prainha, Ilha de Itacuruçá, no Rio de Janeiro, Brasil. As coletas foram realizadas entre os anos de 2014 e 2016 em período sazonal, verão, com o total de 15 coletas 
e perfazendo 4 horas de atividade diariamente, com mergulho livre. Foram identificadas sete espécies: Tegula viridula (GMELIN, 1791); Stramonita haemastoma (LINNAEUS, 1767); Stramanita rustica (LAMARCK, 1822); Neritina virginea (LINNAEUS, 1758); Calliostoma adspersum (PHILIPPI, 1851); Latirus ogum (PETUCH, 1979) e Fissurella clenchi (FARFANTE, 1943). A praia da Viola se mostrou com maior incidência de espécies totalizando 137 espécimes. A espécie T. viridula obteve a maior ocorrência nas duas áreas estudadas. Já L. ogum foi a espécie com menor ocorrência para o estudo.

Palavras-chave: Mollusca, Probranchia, Stramonita haemastoma.

\section{INTRODUÇÃO}

Barbosa (1995) descreve o filo Mollusca como um dos grupos mais diversos em formas e espécies com adaptações funcionais e ambientais específicas para todos os habitats, com as principais classes: Bivalvia, sendo constituída por espécies limitadas ao ambiente aquático; Cephalopoda, espécies exclusivamente marinhas; e Gastrópoda, reunindo o maior número de espécies viventes encontradas em todos os ambientes, tendo atualmente 100 mil espécies.

Os moluscos gastrópodes são animais considerados de grande importância para o ambiente marinho por estarem envolvidos na construção de recifes, por meio de sedimentação de suas conchas mortas, quebradas ou inteiras. São organismos sentinelas e são utilizados como bioindicadores da contaminação por substâncias químicas nos ambientes aquáticos; e possuem hábitos variados de alimentação, podendo ser carnívoros, herbívoros, detritívoros ou suspensores (VILAÇA, 2002; ARAUJO, 2007; CUNHA \& MIYAJI, 2011).

De acordo com Cunha \& Miyaji (2011) a classe Gastropoda está dividida em três subclasses: Prosobranchia, Opstobranchia e Pulmonata. A reprodução desses animais está dividida em gonocórica para os Prosobranchias ou hermafrodita, no caso dos Pulmonatas e Opstobranchias (RUPPERT et al., 2005).

Os costões rochosos sofrem uma grande influência das marés, dividindo-se em zonas supralitoral, médiolitoral e infralitoral, é um ecossistema considerado de grande relevância para a ecologia e economia, por serem ambientes dinâmicos e abrigarem uma grande riqueza de organismos como mexilhões, ostras, crustáceos e uma diversidade de peixes e algas associados (COUTINHO \& ZALMOM, 2009; MORENO \& ROCHA, 2012). São locais de alimentação, crescimento e reprodução de um grande número de espécies, por receber grande quantidade de nutrientes provenientes do ecossistema terrestres, 
apresentando grande biomassa de microfitobentos e de macroalgas, produzindo uma vasta diversidade de organismos, que tornam os costões rochosos um dos mais populares e bem estudados ecossistemas marinhos (COUTINHO et al., 2016).

Ruppert et al., (2005) descrevem os caramujos marinhos como animais adaptados para viver associados ao costão, pois possuem um pé com sola rastejadora, ampla, achatada e com massa muscular que promove sua locomoção, considerado um órgão adesivo onde os caramujos conseguem aderir e viver firme ao substrato; possui glândulas que secretam muco com a finalidade de criar um caminho para que o animal deslize

Wongtschowski \& Amaral (2004), afirmam que as famílias mais abundantes para a região do sudeste brasileira, são Fissurellidae (FLEMING, 1822); Trochidae (RAFINESQUE, 1815); Turbinidae (RAFINESQUE, 1815); Epitoniidae (BERRY, 1910); Muricidae (RAFINESQUE, 1815) e Turridae (SWAINSON, 1840).

O presente estudo tem como justificativa a ausência de trabalhos científicos da malacofauna marinha da Ilha de Itacuruçá, Baía de Sepetiba, Rio de Janeiro, com o objetivo de realizar o levantamento taxonômico da fauna marinha de gastrópodes (caramujos marinhos) na zona infralitoral do costão rochoso das praias da Viola e Prainha.

\section{METODOLOGIA}

As coletas foram realizadas entre os anos de 2014 ao primeiro semestre de 2016 em costões rochosos (zona infralitoral) das praias da Viola (Figura I) e Prainha (Figura II), na Ilha de Itacuruçá localizada na Baía de Sepetiba (2254' $23^{\circ} 04^{\prime}$ S; $43^{\circ} 34^{\prime}-44^{\circ} 10 \mathrm{~W}$ ), no Estado do Rio de Janeiro entre os municípios de Itaguaí e Mangaratiba. Sua área é de aproximadamente 10 $\mathrm{km}^{2}$ e encontra-se inserida na Área de Proteção Ambiental de Mangaratiba (Decreto Federal 9802/1987). Ao total foram realizadas quinze saídas de campo para observação com duração média de quatro horas de atividade cada. Os animais foram observados e registrados com metodologia por fotoquadrado através de mergulho livre, na modalidade apneia, e em profundidades que variavam de $1,0 \mathrm{~m}$ a $2,5 \mathrm{~m}$. Nadadeiras, máscara e snorkel foram utilizados para auxiliar nas coletas. Para a identificação foram coletados somente 42 espécimes dos quais foram observados, para fins de preservação das espécies. Após as coletas, os animais foram armazenados em caixas de polietileno, hermeticamente fechados e transportados para o Laboratório de Biologia Marinha (LabMar), Centro de Pesquisas Biológicas (CEPBio) da Universidade Castelo Branco - RJ. Os animais foram identificados, de acordo com Thomé et al., (2010) e Rios (1994). 4 gotas de formaldeído 4\% $(\mathrm{v} / \mathrm{v})$ foram introduzidas no interior das conchas, e em seguida depositados em frascos de vidro. 


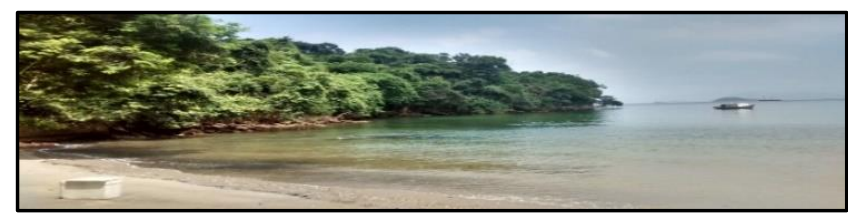

Figura 1. Costão esquerdo da praia da Viola, Ilha de Itacuruçá, RJ. Fonte: acervo pessoal.

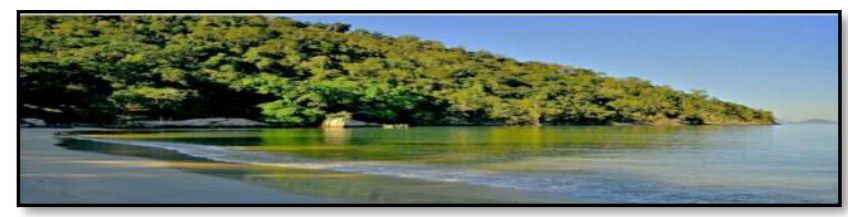

Figura 2. Costão esquerdo da Prainha, Ilha de Itacuruçá, RJ. Fonte: acervo pessoal.

Os costões rochosos esquerdo das duas praias estudadas se mostrou com maior amplitude em relação ao flanco direito, dessa forma foi à área de foco deste estudo. Também foi observado para o ano de 2014 que esses pontos obtinham fatores propícios para a predominância de gastrópodes marinhos, por apresentarem grande cobertura de macroalgas e diversidade de invertebrados.

\section{RESULTADOS E DISCUSSÃO}

Foram identificadas sete espécies de gastrópodes para um $\mathrm{N}$ amostral de 137 animais observados para as praias da Viola e Prainha, como mostra na Tabela 1.

Tabela 1. Lista de espécies de gastrópodes identificados e registrado no Livro de Tombo da Coleção Científica do Laboratório de Biologia Marinha-UCB/RJ.

\begin{tabular}{|c|c|c|}
\hline Espécies & Família & $\begin{array}{l}\mathrm{N}^{\circ} \mathrm{DE} \\
\text { TOMBO }\end{array}$ \\
\hline $\begin{array}{l}\text { Tegula viridula } \\
\text { (Gmelin, 1791) }\end{array}$ & Trochidae & $\begin{array}{l}\text { LABMAR1 } \\
6017\end{array}$ \\
\hline $\begin{array}{l}\text { Stramonita } \\
\text { haemastoma } \\
\text { (Linnaeus, 1767) }\end{array}$ & Muricidae & $\begin{array}{l}\text { LABMAR1 } \\
6005\end{array}$ \\
\hline $\begin{array}{l}\text { Stramonita rustica } \\
\text { (Lamarck, 1822) }\end{array}$ & Muricidae & $\begin{array}{l}\text { LABMAR1 } \\
4006\end{array}$ \\
\hline
\end{tabular}

\begin{tabular}{lll}
\hline $\begin{array}{l}\text { Neritina virgínea } \\
\text { (Linnaeus, 1758) }\end{array}$ & Neritidae & $\begin{array}{l}\text { LABMAR1 } \\
4001\end{array}$ \\
$\begin{array}{l}\text { Calliostoma } \\
\text { adspersum } \\
\text { (Philippi, 1851) }\end{array}$ & $\begin{array}{l}\text { Calliostomat } \\
\text { idae }\end{array}$ & $\begin{array}{l}\text { LABMAR1 } \\
4002\end{array}$ \\
$\begin{array}{l}\text { Latirus ogum } \\
\text { (Petuch, 1979) }\end{array}$ & Fasciolariid & LABMAR \\
& ae & 14003 \\
$\begin{array}{l}\text { Fissurella } \\
\text { clenchi } \\
\text { (Farfante, 1943) }\end{array}$ & Fissurellida & $\begin{array}{l}\text { LABMAR } \\
15009\end{array}$ \\
\hline \hline
\end{tabular}

Fonte: Próprio autor.

O gráfico na Figura 3 demonstra que em sete espécies identificadas, quatro obtiveram maior ocorrência para o ano de 2014. A espécie Tegula viridula ocorreu de forma predominante em todos os anos do estudo nos costões rochosos da área de estudo. Segundo Cabrini \& Cardoso (2012), a reprodução dessa espécie ocorre em todo o ano, com picos ao longo do verão, e que de acordo com Silva (2014), pode estar ligado à variação de temperatura e a disponibilidade de alimento.

A espécie Calliostoma adspersum só foi observado no costão rochoso da praia da Viola no ano de 2014. Este costão se mostrou rico em fauna e flora. Segundo Coutinho \& Zalmon (2009), a alta produtividade de um costão rochoso está relacionada à abundância de nutrientes, onde diversas espécies encontram nesse local a possibilidade de se alimentar, ocasionando assim o crescimento de sua população. 


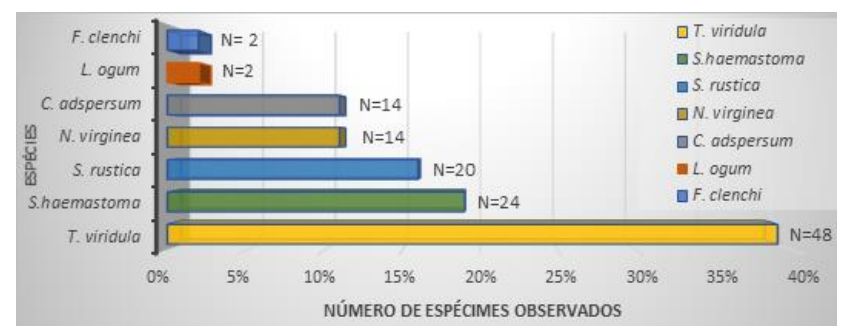

Figura 3. Gráfico de ocorrência de espécies nas praias da Viola e Prainha para o ano de 2014.Fonte: Próprio autor.

No ano de 2015 as espécies Neritina virginea, Latirus ogum e Calliostoma adspersum não tiverem ocorrência para nenhuma das praias, como mostra o gráfico na Figura 4. A espécie Neritina virginea teve sua maior incidência para a Praia da Viola no ano de 2014. Para os outros anos, não foi observado nenhum exemplar. No estudo de Galhardo \& Cardoso (2009), realizado na praia das Flexeiras, na Ilha de Itacuruçá, mostrou que esta espécie foi encontrada em ambientes estuarinos e normalmente em locais com sedimentos lamosos e escuros.

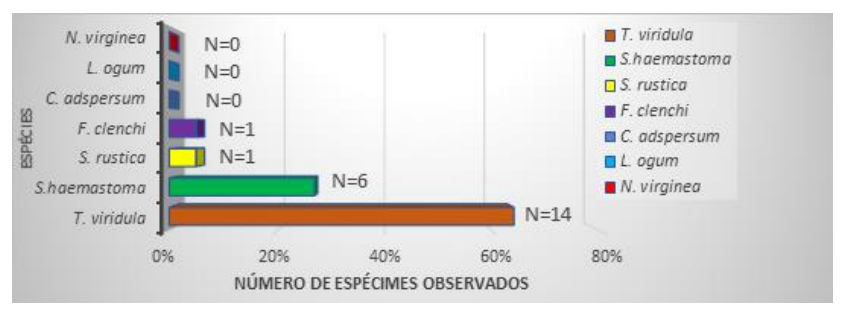

Figura 4. Gráfico de ocorrência de espécies na praias da Viola e Prainha para o ano de 2015. Fonte: Próprio autor.

No primeiro semestre de 2016, notou-se uma queda na ocorrência de animais. Apenas Stramonita haemastoma e Tegula viridula foram observadas na área de estudo, como mostra o gráfico da Figura 6. Os gastrópodes da espécie Stramonita haemastoma foram encontrados em sua maioria na praia da Viola.

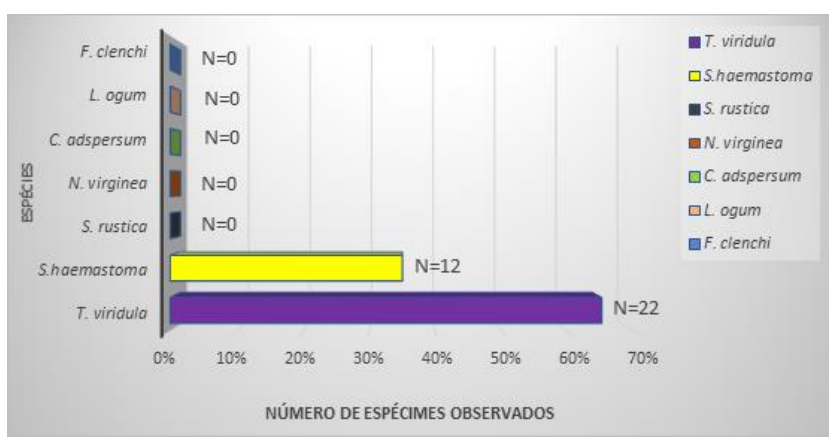

Figura 5. Gráfico de ocorrências de espécies nas praias da Viola e Prainha para o ano de 2016.Fonte: Próprio autor.

Deus et al., (2014) afirmam que para o mês de fevereiro, se registrou uma grande densidade da espécie S. haemastoma também para a Ilha de Palmas da Baía de Santos, e que em um determinado trecho do costão foi encontrado em abundância o mexilhão Perna perna (LINNAEUS, 1758), o que favoreceu a permanência da espécie $S$. haemastoma, pois o mexilhão constitui sua preferência alimentar.

Fissurella clenchi foi a espécie com uma das menores ocorrências para o estudo, com apenas 4 indivíduos coletados sendo 3 para a praia da Viola e 1 para Prainha. Segundo Romitelli (2012), essa espécie se desloca em busca de melhores condições ambientais, porém a limitação fisiológica de $F$. clenchi pode reduzir sua mobilidade na rocha nua. $\mathrm{O}$ que pode explicar o fato de ter sido encontrado apenas 1 espécime para a Prainha onde o costão se encontrou vazio nos anos de 2015 e 2016.

Segundo Cecílio (2012), a herbivoria é realizada diretamente por moluscos, em algas que cobrem o costão. A praia da Viola, foi o local de estudo onde o costão se mostrou com grande 
diversidade de invertebrados e repleto de macroalgas, constituindo a preferência alimentar da maioria das espécies de gastrópodes encontradas.

No costão rochoso da Prainha onde a ocorrência para caramujos marinhos foi menor, a presença de macroalgas foi apenas para o ano de 2014 , e poucos invertebrados foram vistos para a zona infralitoral. Masunari (1987), diz que as macroalgas são de grande importância, funcionando como substrato biológico para diversas espécies sésseis e vágeis, associadas. Parker et al., (2001) comenta que macroalgas disponibilizam-se como habitats diferenciados e possibilitam uma diversidade de alimento e abrigo.

Notou-se uma diminuição nas espécies de caramujos marinhos ao longo dos anos neste estudo. Segundo Vianna (1998), uma hipótese para a diminuição de algas e de organismos bentônicos em pontos da Ilha de Itacuruçá, pode estar associada à contaminação do ambiente e da biota local da Baía de Sepetiba por efluentes industriais e metais pesados.

\section{CONCLUSÃO}

A espécie de gastrópode marinho com maior ocorrência para este estudo foi T. viridula. Esta espécie se mostrou presente no costão das duas praias onde foi realizado o estudo, e também presente em todos as coletas. A espécie L. ogum teve a menor incidência para o estudo. Nos anos de 2015 e 2016 apenas as espécies T. viridula e $S$. haemastoma foram encontradas e não se teve ocorrência das outras três espécies: L. ogum, C. adspersum, $N$. virgínea.

\section{REFERÊNCIAS}

ARAUJO, P. R. P. Moluscos como bioindicadores. Livro de Resumos do XX EBRAM - Rio de Janeiro, 05 a 10 de agosto de 2007. p. 17-18.

BARBOSA, F. S. Tópicos em Malacologia Médica [online]. SciELO Books. p. 1 - 49, 1995.

CABRINI, T. M. B.; CARDOSO, R. S. Population biology of Nassarius vibex (Say, 1822) on a sheltered beach in Southeastern Brazil. J. shellfish Res., v.31, p. 808-815, 2012.

CECÍlLIO, T. M. P. Guia de Identificação Invertebrados Marinhos da Baía de Buarcos. 2012. 97f. Dissertação (Mestrado em Biologia) Faculdade de Ciências E Tecnologia, Universidade de Coimbra, 2012.

COUTINHO, R.; YAGINUMA, L. E.; SIVIERO, F.; SANTOS, J. C. Q. P.; LÓPEZ, M. S.; CHRISTOFOLETTI， R. A.; BERCHEZ， F.; GHILARDI-LOPES, N. P.; FERREIRA, C. E. L.; GONÇALVES, J. E. A.; MASI, B. P.; CORREIA, M. D.; SOVIERZOSKI, H. H.; SKINNER, L. F.; ZALMON, I. R. Studies on benthic communities of rocky shores on the Brazilian coast and climate change monitoring: 
status of knowledge and challenges. Brazilian Journal of Oceanography, 64 (sp2): 27-36; 2016.

COUTINHO, R. C. \& ZALMON, I. R. Os Bentos de Costões Rochosos. In PEREIRA, R.C; GOMES, A.S (Ed). Biologia Marinha. $2^{\circ}$ ed, Rio de Janeiro, Ed. Interciência, p. 281-298. 2009.

CUNHA, C. M. \& MIYAJI, C. Gastropoda. In AMARAL, A. C. Z.; NALLIN, S. A. H. Biodiversidade e Ecossistemas Bentônicos Marinhos do Litoral Norte de São Paulo Sudeste do Brasil. Ficha Catalográfica Elaborada pela Biblioteca Central da UNICAMP. Campinas/ SP. p. 228-235, 2011.

DEUS, S. R. F.; COSTA, J. A.; MOTTA, N. S.; CASARINI, L. M. Estimativa da Densidade de Stramonita haemastoma em Recifes Rochosos na Baía de Santos. Revista Ceciliana. v.6, n. 2, p. 2427, 2014.

GALHARDO, L. B. \& CARDOSO, R. S. Padrões de coloração de Neritina virginea encontrados na praia das Flexeiras, Ilha de Itacuruçá, Rio de Janeiro, Brasil. XXI Encontro Brasileiro De Malacologia. p. 314, 2009.

MASUNARI, S. Ecologia das Comunidades Fitais. Academia de Ciências do Estado de São Paulo. Simpósio Sobre Ecossistemas da Costa Sul e Sudeste Brasileira. p. 459, 1987.
MORENO, T. R. \& ROCHA, R. M. Ecologia de costões rochosos. Estud. Biol., Ambiente Divers, Paraná, v.34, n.83, p. 191-201, 2012.

PARKER, J. D.; DUFFY, E.; ORTH, R. J. Plant species diversity and composition: experimental effects on marine epifaunal assemblages. Mar. Ecol. n. 224, p. 55-67, 2001.

RIOS, E. C. SEASHELLS OF BRAZIL. $2^{\circ} \mathrm{ed}$, Rio Grande, RS. Ed. da Furg. p. 133 , 1994.

ROMITELLI, I. Permeabilidade de duas espécies de lapa (Mollusca: Gastropoda) em matriz de rocha nua em costões. Pratica da pesquisa em Ecologia da Mata Atlântica. Curso de Pósgraduação em Ecologia - Universidade de São Paulo, 2012.

RUPPERT, E. E.; FOX, R. S.; BARNES, R. D. Zoologia de Invertebrados. $7^{\circ}$ ed, São Paulo. Ed. Roca, p. 1168, 2005.

SILVA, V. F. Dinâmica populacional do caramujo marinho Tegula viridula (GMELIN, 1791) utilizando dois tipos de dados: marcação e recaptura e distribuição de frequências. 2014. 53f. Dissertação (Pós-graduação em Ciências Biológicas), Instituto de Biociências do Centro de Ciências Biológicas e da Saúde da Universidade Federal Do Estado do Rio de Janeiro, 2014.

THOMÉ, J. W.; TARASCONI, J. C.; BERGONCI, P. E. A.; GIL, G. As Conchas Das Nossas Praias. $2^{\circ}$ ed, Ed. Redes, p. 223, 2010. 
VIANNA, L. F. N; Diagnóstico Sócio Ambiental da Ilha de Itacuruçá, Mangaratiba / Itaguaí, RT - Brasil, Subsidiário à Estruturação Do Programa De Gestão Costeira Integrada. 1998. 241f. Dissertação (Pós-Graduação em Engenharia Ambiental), Departamento de Engenharia Sanitária e Ambiental, Universidade Federal de Santa Catarina. Florianópolis, 1998.

VILLAÇA, R. Recifes biológicos. In Biologia Marinha. Pereira \& Gomes. Ed. Interciência. Rio de Janeiro. p. 229248, 2002.
WONGTSCHOWSKI, C. L. D. B. R. \& AMARAL, C. Z A. Biodiversidade Bentônica da Região Sudeste-Sul do Brasil - Plataforma Externa e Talude Superior. REVIZEE BENTOS. p. 1-216, 2004.

Mass Spectrom 35:1246-1251. 